\title{
An Analytical Study of Smooth Solutions of the Bløtekjær Hydrodynamic Model of Electron Transport
}

\author{
JOSEPH W. JEROME* \\ Department of Mathematics, Northwestern University, Evanston, IL 60208, USA
}

(Received 1 May 2001; Revised 1 April 2002)

\begin{abstract}
We study the Bløtekjær hydrodynamic model from the standpoint of local well-posedness. We employ analytical methods, originally introduced by T. Kato for complex systems, to obtain the existence of unique local smooth solutions of the Cauchy problem, with smooth initial data. The time interval is invariant with respect to vanishing heat flux. The model is self-consistent, and is developed for onevalley electron carriers only. A symmetrizer is introduced for the system, and regularization is employed to avoid the formation of singularities due to vacuum regions. In the regime studied, it is not possible for shocks to form.
\end{abstract}

Keywords: Bløtekjær model; Cauchy problem; Wiedemann-Franz law; Smooth solutions; Vanishing heat flux limit; Semigroup generators

\section{INTRODUCTION}

\section{The Hydrodynamic Bløtekjær Model}

The equations as presented here were introduced in Ref. [1], developed in Ref. [2] and discussed at length in Ref. [3]. They are derived as the first three moments of the Boltzmann transport equation,

$$
\frac{\partial f}{\partial t}+\mathbf{u} \cdot \nabla_{x} f-\frac{e}{m} \mathbf{E} \cdot \nabla_{u} f=C .
$$

Here, $f=f(x, \mathbf{u}, t)$ is the numerical distribution function of the electron species, $\mathbf{u}$ is the species' group velocity vector, $\mathbf{E}=\mathbf{E}(x, t)$ is the electric field, $e$ is the electron charge modulus, $m$ is the effective electron mass, and $C$ is the time rate of change of $f$ due to collisions. The moment equations are expressed in conservation form in terms of certain "conserved" dependent variable quantities: the electron concentration, $n$; the momentum density, $\mathbf{p}$; and the energy density, $w$. The concentration is given formally by $n:=\int f \mathrm{~d} \mathbf{u}$; the average velocity by $\mathbf{v}:=(1 / n) \int \mathbf{u} f \mathrm{~d} \mathbf{u}$; and the momentum by $\mathbf{p}:=m n \mathbf{v}$. The selection of $w$, as well as the symmetric pressure tensor, $\mathbf{P}$, and the the heat flux, $\mathbf{q}$, is part of the set of moment closure relationships. Additionally, $e_{\mathrm{I}}$ is the internal energy, and if $C_{n}, C_{p}$ and $C_{w}$ represent moments of $C$, taken with respect to the functions, $h_{0}(\mathbf{u}) \equiv 1, \quad h_{1}(\mathbf{u})=m \mathbf{u}, \quad h_{2}(\mathbf{u})=(m / 2)|\mathbf{u}|^{2}$, then the hydrodynamic equations of the Bløtekjær model are:

$$
\begin{gathered}
\frac{\partial n}{\partial t}+\nabla \cdot(n \mathbf{v})=C_{n}, \\
\frac{\partial \mathbf{p}}{\partial t}+\mathbf{v} \nabla \cdot \mathbf{p}+(\mathbf{p} \cdot \nabla) \mathbf{v}+\nabla \cdot \mathbf{P}=-e n \mathbf{E}+C_{\mathbf{p}}, \\
\frac{\partial w}{\partial t}+\nabla \cdot(\mathbf{v} w)+\nabla \cdot(\mathbf{P v})+\nabla \cdot \mathbf{q}=-e n \mathbf{v} \cdot \mathbf{E}+C_{w} .
\end{gathered}
$$

Poisson's electrostatic equation for the electric potential must be adjoined, where $n_{\mathrm{d}}:=$ doping and $\epsilon:=$ dielectric :

$$
\begin{gathered}
\mathbf{E}=-\nabla \phi, \\
\nabla \cdot(\epsilon \nabla \phi)=e n-n_{\mathrm{d}} .
\end{gathered}
$$

The entire system consists of Eqs. (1.2)-(1.6). By moment closure is meant the selection of compatible relations for the variables, $n, \mathbf{v}, \mathbf{P}, w$ and $\mathbf{q}$. We make the isotropy assumption:

$$
P_{i j}=P \delta_{i j}
$$

*Tel.: +1-847-491-5575. Fax.: + 1-847-491-8906. E-mail: jwj@math.nwu.edu 
for a scalar pressure $P$, and introduce a new variable $\mathscr{T}$, the carrier temperature, defined by $P=n k \mathscr{T}$, where $k$ is Boltzmann's constant. The Bløtekjær model then makes the following selections for the closure relations.

1. The internal energy is expressed by $m e_{\mathrm{I}}=(3 / 2) \mathrm{k} \mathscr{T}$.

2. The total energy density (per unit volume) $w$ is given by combining internal energy and parabolic energy bands with $m$ assumed constant:

$$
w=m n e_{\mathrm{I}}+\frac{1}{2} m n|\mathbf{v}|^{2} .
$$

3. The heat flux is obtained by the Fourier law of heat conduction (cf. Eq. (1.7) to follow).

The final step deals with the replacement of the collision moments. For a one carrier system, we define $C_{n}=0$, and appropriate momentum and energy relaxation expressions. We have:

1. The momentum relaxation approximation is given via

$$
-\frac{\mathbf{p}}{\tau_{\mathbf{p}}}:=m \int \mathbf{u} C \mathrm{~d} \mathbf{u}:=\mathrm{C}_{\mathbf{p}}
$$

2. The energy relaxation approximation is given via

$$
-\frac{n\left(m e_{\mathrm{I}}-w_{0}\right)}{\tau_{w}}-\frac{m n|\mathbf{v}|^{2}}{2 \tau_{w}}:=\frac{m}{2} \int|\mathbf{u}|^{2} C \mathrm{~d} \mathbf{u}:=C_{w} .
$$

Here, $w_{0}$ denotes the rest energy, $(3 / 2) k \mathscr{T}_{*}$, where $\mathscr{T}_{*}$ is the lattice temperature.

The forms for the relaxation times often used for simulation are the Baccarani-Wordeman models [4]. In this analytical study, we shall employ simplified versions, taking these quantities to be constant. In the concluding remarks, we shall indicate the easy modifications required to deal with the general temperature-dependent case.

The traditional form of $\mathbf{q}$ has been (see Ref. [5] for more general expressions),

$$
\mathbf{q}=-\kappa \nabla \mathscr{T}
$$

Here, $\kappa$ is the thermal conductivity. In simulation, this is often selected according to the Wiedemann-Franz (WF) law [6]. This involves proportionality of the conductivity to the concentration $n$, which introduces technical issues. However, our approach is sufficiently robust that we can deal with such a variable conductivity.

Dependence on $n$ : We shall assume for the body of the analysis to follow that $\kappa$ is of the form,

$$
\kappa=\kappa_{0} n,
$$

where $\kappa_{0}$ is a fixed positive parameter. The theory of this paper will establish stability under the limit $\kappa_{0} \rightarrow 0$.

\section{System Reformulation in Nonconservative Form}

We reformulate the system (1.2)-(1.4) in nonconservative form via the dependent variables $n, \mathbf{v}$ and $\mathscr{T}$. Elementary multidimensional differential calculus gives the equivalent system:

$$
\begin{gathered}
\frac{\partial n}{\partial t}+\mathbf{v} \cdot \nabla n+n \nabla \cdot \mathbf{v}=0 \\
\frac{\partial \mathbf{v}}{\partial t}+(\mathbf{v} \cdot \nabla) \mathbf{v}+\frac{k}{m} \nabla \mathscr{T}+\frac{k \mathscr{T}}{m n} \nabla n=-\frac{e}{m} E-\frac{\mathbf{v}}{\tau_{\mathbf{p}}}, \\
\frac{\partial \mathscr{T}}{\partial t}-\frac{\kappa_{0}}{n} \nabla \cdot(n \nabla \mathscr{T})+\mathbf{v} \cdot \nabla \mathscr{T}+\frac{2}{3} \mathscr{T} \nabla \cdot \mathbf{v} \\
=-\frac{2 m|\mathbf{v}|^{2}}{3 k}\left(\frac{1}{2 \tau_{w}}-\frac{1}{\tau_{\mathbf{p}}}\right)-\frac{\mathscr{T}-\mathscr{T} *}{\tau_{w}} .
\end{gathered}
$$

In the language of Friedrichs, there is a symmetrizer for the system. We shall consider the problem on Euclidean space of dimension $d$. Define the $d+2$-vector $\mathbf{u}$ by

$$
\mathbf{u}=\left[\begin{array}{c}
n \\
\mathbf{v} \\
\mathscr{T}
\end{array}\right]
$$

The system as defined above has matrix multipliers of $\partial \mathbf{u} / \partial x_{j}$ given by the order $d+2$ matrices:

$$
\tilde{A}_{j}=\left[\begin{array}{c|c|c}
v_{j} & n, \ldots, n & 0 \\
\hline \frac{k \mathcal{T}}{m n} & v_{j}, \ldots, v_{j} & \frac{k}{m} \\
\ldots & \ldots & \ldots \\
\frac{k \mathcal{T}}{m n} & v_{j}, \ldots, v_{j} & \frac{k}{m} \\
\hline 0 & \frac{2 \mathcal{T}}{3}, \ldots, \frac{2 \mathcal{T}}{3} & v_{j}
\end{array}\right]
$$

The symmetrizer is then given by:

$$
A_{0}=\left[\begin{array}{c|c|c}
\frac{k T}{m n} & 0 & 0 \\
\hline 0 & n I_{d} & 0 \\
\hline 0 & 0 & \frac{3 k n}{2 m T}
\end{array}\right]
$$

$A_{0}$ is symmetrizing in the following sense:

$$
A_{j}=A_{0} \tilde{A}_{j}=\left[\begin{array}{c|c|c}
\frac{k T v_{j}}{m n} & \frac{k \mathcal{T}}{m}, \ldots, \frac{k \mathcal{T}}{m} & 0 \\
\hline \frac{k \mathcal{T}}{m} & n v_{j}, \ldots, n v_{j} & \frac{k n}{m} \\
\frac{k \mathcal{T}}{m} & n v_{j}, \ldots, n v_{j} & \frac{k n}{m} \\
\hline 0 & \frac{k n}{m}, \ldots, \frac{k n}{m} & \frac{3 k n v_{j}}{2 m \mathcal{T}}
\end{array}\right]
$$

is symmetric for each $j=1, \ldots, d$. We shall select units in which $k / m=1$ and $e / m=1$. We may finally rewrite the 
system, after its symmetrization, as

$$
a_{0}(\mathbf{u}) \mathbf{u}_{t}+L(\mathbf{u}) \mathbf{u}+\left[\sum_{j=1}^{d} a_{j}(\mathbf{u}) \frac{\partial \mathbf{u}}{\partial x_{j}}+b(\mathbf{u}) \mathbf{u}\right]=0
$$

where

$$
\begin{aligned}
& a_{0}=\left[\begin{array}{c|c|c}
\frac{\mathcal{T}}{n} & 0 & 0 \\
\hline 0 & n I_{d} & \mathbf{0} \\
\hline 0 & \mathbf{0} & \frac{3 n}{2 \mathcal{T}}
\end{array}\right], a_{j}=\left[\begin{array}{c|c|c}
\frac{\mathcal{T} v_{j}}{n} & \mathcal{T}, \ldots, \mathcal{T} & 0 \\
\hline \mathcal{T} & n v_{j}, \ldots, n v_{j} & n \\
\ldots & \ldots & \ldots \\
\mathcal{T} & n v_{j}, \ldots, n v_{j} & n \\
\hline 0 & n, \ldots, n & \frac{3 n v_{j}}{2 \mathcal{T}}
\end{array}\right], \\
& b=\left[\begin{array}{c|c|c}
0 & 0 & 0 \\
\hline-(\nabla \phi)^{T} & \frac{n}{\tau_{\mathrm{p}}} I_{d} & \mathbf{0} \\
\hline \frac{3\left(1-\frac{T_{*}}{\mathcal{T}}\right)}{2 \tau_{w}} & c \frac{n \mathbf{v}}{\mathcal{T}} & 0
\end{array}\right],
\end{aligned}
$$

and where

$$
\begin{aligned}
L(\mathbf{u}) & =-\operatorname{diag}\left(0, \mathbf{0}, \gamma_{0} / \mathscr{T}\right) \nabla \cdot(n \nabla), \quad \gamma_{0}=\frac{3}{2} \kappa_{0}, \\
c & =\left(\frac{1}{2 \tau_{w}}-\frac{1}{\tau_{\mathbf{p}}}\right) .
\end{aligned}
$$

In the above system, the function $\phi$ has been used implicitly in its dependence upon $n$. We make this explicit:

$$
\phi=\Phi\left(u_{1}\right), \quad \text { where }-\epsilon \nabla^{2} \phi=-e u_{1}+n_{\mathrm{d}} .
$$

The assumptions on the smoothing map $\Phi$ are specified later. The initial condition for the Cauchy problem is given by,

$$
\mathbf{u}(\cdot, 0)=\mathbf{u}_{0},
$$

for a given function, $\mathbf{u}_{0} \in H^{\mathrm{s}}\left(R^{d} ; R^{d+2}\right), s>d / 2+2$ (see Eq. (3.2) for positivity conditions). The spaces $H^{\mathrm{s}}$ are defined in "A framework for analysis" section to follow. The complete Cauchy problem is defined by, Eqs. (1.15), (1.17) and (1.18). We shall pose it as a Cauchy problem in Hilbert space. The major result of the paper is Theorem 4.2 .

\section{THE GENERAL INITIAL-VALUE PROBLEM}

\section{Preliminaries}

We begin by describing a class of operators related to the infinitesimal generators of semigroups. If desired, the reader may proceed directly to the next section, "Properties of the Bløtekjær system".

Definition 2.1 Let $U$ be a closed linear operator with domain and range dense in a Hilbert space $X$. Denote by $R(\lambda, U)$ the resolvent $(\lambda I-U)^{-1}$ for $\lambda$ in the resolvent set $\rho(U)$. For $M>0$ and $\omega \in R$ denote by $G(X, M, \omega)$ the set of all operators $A=-U$ such that

$$
\left\|[R(\lambda, U)]^{r}\right\| \leq M(\lambda-\omega)^{-r}, \quad r \geq 1, \quad \lambda>\omega .
$$

Finally,

$$
G(X)=\cup_{\omega, M} G(X, M, \omega)
$$

There is a criterion due to Kato [7,8], which permits one to deduce semigroup generation on a smooth space $Y$ via stability on $X$. More precisely, the criterion is directed more fundamentally at transferring the property $A \in G(X, M, \omega)$ to $A \in G\left(Y, M, \omega_{1}\right)$. It is particularly useful when $M=1$. We quote the relevant result. This result follows from Ref. [8, Propositions 6.2.3 and 6.2.4], which are based on Kato's work [7].

Proposition 2.1 Suppose $Y$ is a Hilbert space densely and continuously embedded in $X$ and $S: Y \mapsto X$ is an isomorphism. We write $\|v\|_{Y}=\|S v\|_{X}$. Suppose $A \in G(X, M, \omega)$ such that

$$
A_{1}=S A S^{-1}=A+B
$$

where $B$ is a bounded linear operator on $X$ and

$$
\mathscr{D}\left(A_{1}\right)=\left\{v: A S^{-1} v \in Y\right\} .
$$

Then the semigroup generated by $-A$, restricted to $Y$, is the semigroup generated by the restriction of $-A$ to $\{v \in Y \cap \mathscr{D}(A): A v \in Y\}$. In fact, $S e^{-t A} S^{-1}=e^{-t A_{1}}$ holds. It follows that $A_{1} \in G(X, M, \omega+M\|B\|)$ or equivalently $A \in G\left(Y, M, \omega_{1}\right)$, with $\omega_{1}=\omega+M\|B\|$.

In order to make use of this theorem, we need to know a fundamental fact about ground space perturbation by a bounded linear operator. We quote the result [9, Theorem 2.1, p. 497]

Proposition 2.2 Let $A$ be in $G(X, M, \omega)$, and let $B$ be bounded on $X$. Then

$$
A+B \in G(X, M, \omega+M\|B\|) .
$$

\section{The Abstract Cauchy Problem}

We are interested in solving an initial value problem, in a Hilbert space $X$,

$$
A_{0}(t, u) \frac{\mathrm{d} u}{\mathrm{~d} t}+A(t, u) u=0, \quad u(0)=u_{0},
$$

where $A(t, u) \in G(X, M, \omega)$ for $u$ restricted to a subset of a "smooth" Hilbert space $Y$, densely and continuously embedded in $X$, and where $A_{0}$ and $A_{0}^{-1}$ are bounded on $X$ for $u$ suitably restricted. We seek a solution $u(t) \in Y$, $0 \leq t \leq T$. The derivative, $\mathrm{d} u / \mathrm{d} t$, is required to belong to an intermediate space, $V$. Certain Lipschitz continuity conditions, to be described later, are also required. Among 
these, we require:

$$
\left\|A_{0}^{-1}(t, v) A(t, u)-A_{0}^{-1}(t, v) A(t, w)\right\|_{Y, X} \leq C\|u-w\|_{X},
$$

uniformly in $v$, for $u, w$ suitably restricted in norm.

\section{The Implicit Semidiscretization in Time}

If $\Delta t$ is given as the ratio $T / N$, then the method of horizontal lines, applied to Eq. (2.2), yields a semidiscrete set of implicit equations, explicit in $A_{0}$ :

$$
\begin{aligned}
& A\left(t_{k}, u_{k}^{N}\right) u_{k}^{N}+(1 / \Delta t) A_{0}\left(t_{k-1}, u_{k-1}^{N}\right) u_{k}^{N} \\
& \quad=(1 / \Delta t) A_{0}\left(t_{k-1}, u_{k-1}^{N}\right) u_{k-1}^{N}, \\
& \quad k=1, \ldots, N .
\end{aligned}
$$

If we set $\mu^{2}=1 / \Delta t=N / T$, then the $u_{k}^{N}$ can be characterized formally as fixed points of the mapping,

$$
\begin{aligned}
Q v= & Q_{k}^{N} v \\
= & -R\left(\mu^{2}-1,-A_{0}^{-1}\left(t_{k-1}, u_{k-1}^{N}\right) A\left(t_{k}, v\right)\right) v \\
& +\mu^{2} R\left(\mu^{2}\right. \\
& \left.-1,-A_{0}^{-1}\left(t_{k-1}, u_{k-1}^{N}\right) A\left(t_{k}, v\right)\right) u_{k-1}^{N} .
\end{aligned}
$$

By repeated back substitution, one obtains the following useful formula for $u_{k-1}^{N}$ :

$$
u_{k-1}^{N}=\prod_{j=1}^{k-1} \mu^{2} R\left(\mu^{2},-\tilde{A}\left(t_{j}, u_{j}^{N}\right)\right) u_{0}
$$

where we have written,

$$
\tilde{A}\left(t_{j}, u_{j}^{N}\right)=A_{0}^{-1}\left(t_{j-1}^{N}, u_{j-1}^{N}\right) A\left(t_{j}, u_{j}^{N}\right)
$$

Pivotal to the entire study is the demonstration of the existence of fixed points for this map within an appropriately smooth ball. The concept of stability proves useful. We are now able to introduce the type of stability which is appropriate.

Definition 2.2 Let $X$ be a Hilbert space, suppose that $\Delta t=T / N$ is given, and a partition $t_{j}=j \Delta t, j=0, \ldots, N$, is specified. Suppose that a family $\{\tilde{A}(t, u)\}$ is given as above, and, for $1 \leq k \leq N$, that $u_{1}^{N}, \ldots, u_{k-1}^{N}$ are arbitrary in $X$. Define $\tilde{A}\left(t_{j}\right)=\tilde{A}\left(t_{j}, u_{j}^{N}\right), j=1, \ldots, k-1$, and $\tilde{A}\left(t_{k}\right)=\tilde{A}\left(t_{k}, u\right)$, for arbitrary fixed $u$. The family is said to be stable if there are constants $M, \omega$ and $c$, independent of $k$ and $N$, such that

$$
\left\|\prod_{j=1}^{k}\left[\tilde{A}\left(t_{j}\right)+\lambda\right]^{-1}\right\| \leq M(\lambda-\omega)^{-k} \exp (c T),
$$

$\lambda>\omega$.
We shall require stability on $X$ and $Y$, with constants $M$, $\omega, c$ and $M_{1}, \omega_{1}, c_{1}$, respectively.

\section{The Fixed Point Theorem}

For $\omega$ and $\omega_{1}$ introduced through Definition 2.2 above, we define: $\bar{\omega}=\max \left(\omega, \omega_{1}\right) . \quad \bar{M}$ and $\bar{c}$ are defined similarly. Suppose that $\delta$ and $\rho$ are fixed positive constants, and that

$$
\sigma=(1+\delta) \bar{M} e^{(1+1 / \rho)(1+\bar{\omega}+\bar{c}) T}
$$

where $T$ is a fixed terminal time to be specified. We define

$$
\bar{W}_{0}=\left\{u \in Y:\|u\|_{Y} \leq \sigma\left\|u_{0}\right\|_{Y},\|u\|_{X} \leq \sigma\left\|u_{0}\right\|_{X}\right\} .
$$

Proposition 2.3 Suppose that a family $\{\tilde{A}(t, u)\}$ and $\Delta t=T / N$ are given, and a partition $t_{j}=j \Delta t, j=0, \ldots, N$, is thereby specified. If $u_{1}^{N}, \ldots, u_{k-1}^{N} \in \bar{W}_{0}$ are inductively defined solutions of Eq. (2.3), suppose that the family $\left\{\tilde{A}\left(t_{j}\right)\right\}$ is defined as in Definition 2.2 and is stable on $X$ and $Y$. If the integer $N$ satisfies:

$$
\frac{N}{T}>\left[\left(1+\delta^{-1}\right) \bar{M}+(\rho+1)(1+\bar{\omega}+\bar{c})\right],
$$

then the mappings $Q=Q_{k}^{N}$ of Eq. (2.4) are mappings of $\bar{W}_{0}$ into itself.

Proof Thus, for fixed $N$, we assume inductively that Eq. (2.3) has a solution $u_{\ell}^{N}$ for $\ell<k$, where $1 \leq k \leq N$. We can estimate $\|Q v\|_{X}$. From Eq. (2.4), we have, by use of the stability property:

$$
\begin{aligned}
\|Q v\|_{X} \leq & \frac{M}{\mu^{2}-\omega-1} \sigma\left\|u_{0}\right\|_{X} \\
& +\frac{M \mu^{2}}{\mu^{2}-\omega-1}\left(\frac{\mu^{2}}{\mu^{2}-\omega}\right)^{k-1} \exp (c T)\left\|u_{0}\right\|_{X} .
\end{aligned}
$$

Here we have used Eq. (2.5). For the first term, we estimate, by the choice of $N$,

$$
\frac{M}{\mu^{2}-\omega-1} \leq \frac{\delta}{(1+\delta)}
$$

An observation required for the estimate of the second term is given by

$$
\frac{\mu^{2}}{\mu^{2}-\omega-1} \leq(1+1 / \rho)
$$

if $\mu^{2} \geq(1+\rho)(\omega+1)$. When this is combined with the standard inequality,

$$
(1+s)^{N} \leq e^{s N}, \quad s=\frac{\omega+1}{\mu^{2}-\omega-1},
$$


we arrive at a chain of inequalities for the second term. By the choice of $N$ and $\sigma$,

$$
\begin{aligned}
& \frac{\mu^{2}}{\mu^{2}-\omega-1}\left(\frac{\mu^{2}}{\mu^{2}-\omega}\right)^{k-1} \exp (c T) \\
& \leq\left(\frac{\mu^{2}}{\mu^{2}-\omega-1}\right)^{N} \exp (c T) \leq e^{(1+1 / \rho)(1+\omega+c) T} \\
& \leq \frac{\sigma}{M(1+\delta)}
\end{aligned}
$$

If we apply each of the estimates, we have the estimate that $\|Q v\|_{X} \leq \sigma\left\|u_{0}\right\|_{X}$.

The estimate for $\|Q v\|_{Y}$ is similar and completes the proof.

\section{Lipschitz Continuity of $Q$}

We shall next establish Lipschitz continuity of $Q$. This will close the induction, and give the existence of $u_{k}^{N}$, for $\Delta t$ sufficiently small.

Proposition 2.4 Under the assumptions of Proposition 2.3, the mappings $Q=Q_{k}^{N}$ of Eq. (2.4) are Lipschitz continuous mappings in the topology of $X$ with Lipschitz constant,

$$
\begin{aligned}
C_{Q}= & \frac{\bar{M}}{\mu^{2}-1-\bar{\omega}}[1+C(1+\bar{M}(1 \\
& \left.+1 / \rho)) \sigma\left\|u_{0}\right\|_{X}\right] .
\end{aligned}
$$

Here, $C$ is the Lipschitz constant cited earlier. If $N$ is sufficiently large, then $C_{Q}<1$ and $Q$ has a unique fixed point in $\bar{W}_{0}$.

Proof The critical representation is the identity,

$$
\begin{aligned}
& R(\lambda,-\tilde{A}(t, w))-R(\lambda,-\tilde{A}(t, v)) \\
& \quad=R(\lambda,-\tilde{A}(t, w))[\tilde{A}(t, v)-\tilde{A}(t, w)] R(\lambda,-\tilde{A}(t, v))
\end{aligned}
$$

We obtain:

$$
\begin{aligned}
& \|R(\lambda,-\tilde{A}(t, w))-R(\lambda,-\tilde{A}(t, v))\|_{X} \\
& \leq\|R(\lambda,-\tilde{A}(t, w))\|_{X} C_{\tilde{A}}\|v-w\|_{X}\|R(\lambda,-\tilde{A}(t, v))\|_{Y} \\
& \quad \leq C_{1} \frac{\|v-w\|_{X}}{(\lambda-\omega)\left(\lambda-\omega_{1}\right)}
\end{aligned}
$$

where $C_{1}=M M_{1} C_{\tilde{A}}$. This leads to the estimate, for $\lambda=\mu^{2}-1$,

$$
\begin{aligned}
& \|Q v-Q w\|_{X} \leq \frac{1}{\mu^{2}-1-\omega} \\
& \quad \times\left[M+\frac{C_{1} \sigma\left\|u_{0}\right\|_{X}}{\mu^{2}-1-\omega_{1}}+\frac{C_{1} \sigma\left\|u_{0}\right\|_{X} \mu^{2}}{\mu^{2}-1-\omega_{1}}\right]\|v-w\|_{X} .
\end{aligned}
$$

Here, we have used the inductive assumption that $\left\|u_{k-1}^{N}\right\|_{X} \leq \sigma\left\|u_{0}\right\|_{X}$. By using the estimates of the proof of Proposition 2.3, we obtain

$$
\begin{aligned}
& \|Q v-Q w\|_{X} \leq \frac{1}{\mu^{2}-1-\omega} \\
& {\left[M+\frac{M C_{\tilde{A}} \sigma \delta\left\|u_{0}\right\|_{X}}{(1+\delta)}+M M_{1} C_{\tilde{A}}(1+1 / \rho) \sigma\left\|u_{0}\right\|_{X}\right]} \\
& \|v-w\|_{X} .
\end{aligned}
$$

This yields the estimate (2.11) of the proposition. Since $Y$ is assumed to be an embedded Hilbert space, $\bar{W}_{0}$ is a complete metric subspace of $X$, and the final statement follows from the contraction mapping theorem [10 Theorem 1, pp. 214-215].

\section{Conditions for Stability}

We investigate natural conditions for stability. We shall first recall the notion for families $\{\tilde{A}(t)\}$ defined for each $0 \leq t \leq T$. By stability for such a family, we mean (2.6) for each subset $\left\{t_{j}\right\}$ of $[0, T]$. We have the following proposition for such a family, quoted from Ref. [8 Proposition 6.2.2].

Proposition 2.5 For each $t \in[0, T]$, let $\|\cdot\|_{t}$ be a new norm in $X$, let $X_{t}=\left(X,\|\cdot\|_{t}\right)$, and suppose there is a real number $c_{0}$ such that

$$
\|f\|_{t} /\|f\|_{s} \leq e^{c_{0}|t-s|}, \quad f \in X, \quad s, t \in[0, T]
$$

Suppose $\tilde{A}(t) \in G\left(X_{t}, 1, \omega_{0}\right), \forall t \in[0, T]$. Then $\{\tilde{A}(t)\}$ is stable, with $M=1, c=2 c_{0}, \omega=\omega_{0}$, with respect to $X_{t}$, for each $t \in[0, T]$.

Since we shall not require the full strength of this notion of stability, we formulate the discrete version to be used later. The proof is the same as that of Proposition 2.5.

Proposition 2.6 Let a uniform partition $\left\{t_{j}\right\}$ of $[0, T]$ be specified, and for each $t \in\left\{t_{j}\right\}$ let $X_{t}=\left(X,\|\cdot\|_{t}\right)$ be specified. If (2.12) holds for $s, t \in\left\{t_{j}\right\}$, then the conclusion of Proposition 2.5 holds in the restricted sense, for $t \in\left\{t_{j}\right\}$.

Suppose we have the setup of Proposition 2.6, and suppose $Y \subset X$ are related by an isomorphism, $S: Y \mapsto X$, and that $S$ remains an isomorphism from $Y_{t}$ to $X_{t}$, where

$$
\|u\|_{Y_{t}}=\|S u\|_{X_{t}}, \quad t \in\left\{t_{j}\right\} .
$$

We can deduce stability on $Y$ from stability on $X$ by a routine application of Proposition 2.1 to Proposition 2.6. We have the following.

Proposition 2.7 For each $t \in\left\{t_{j}\right\}$, let $\left(X,\|\cdot\|_{t}\right)$ be defined as in Proposition 2.6, where the condition (2.12) is 
assumed to hold. Suppose Eq. (2.1) holds for each member of the family $\{\tilde{A}(t)\}$ :

$$
\tilde{A}_{1}(t)=S \tilde{A}(t) S^{-1}=\tilde{A}(t)+\tilde{B}(t),
$$

where $\|\tilde{B}(t)\|_{X}$ is uniformly bounded. If $\tilde{A}_{1}(t) \in$ $G\left(X_{t}, 1, \omega\right), \forall t \in\left\{t_{j}\right\}$, then $\{\tilde{A}(t)\}$ is stable, with $M_{1}=1, c_{1}=2 c_{0}, \omega_{1}=\omega+\|B(t)\|_{t}$, with respect to $Y_{t}$, for each $t \in\left\{t_{j}\right\}$.

\section{PROPERTIES OF THE BLøTEKJæR SYSTEM}

It is necessary to regularize the symmetrizer $a_{0}$, given in Eq. (1.16), as well as other multipliers to prevent the formation of singularities. We will work with the regularized problem, and in the final section indicate that this implies the local result for the non-regularized problem.

\section{The Regularization of $a_{0}, a_{j}$ and $L$}

Let $\zeta$ be a non-decreasing $C^{\infty}$ function satisfying:

$$
\zeta(s)= \begin{cases}0, & s \leq 0 \\ 1, & s \geq 1 .\end{cases}
$$

An example of such a function is given in Ref. [11, p. 36] Given the initial conditions, $n_{0}, \mathscr{T}_{0}$, and constant threshold values,

$$
0<n_{00}<\inf n_{0}, 0<\mathscr{T}_{00}<\inf \mathscr{T}_{0},
$$

define the regularizations by setting

$$
\begin{aligned}
& \tilde{n}(n)=\frac{n}{2}\left(1+\zeta\left(n / n_{00}\right)\right), \\
& \tilde{\mathscr{T}}(\mathscr{T})=\frac{\mathscr{T}}{2}\left(1+\zeta\left(\mathscr{T} / \mathscr{T}_{00}\right)\right) .
\end{aligned}
$$

Then, $\tilde{n}(n)=n, n \geq n_{00}, \tilde{\mathscr{T}}(\mathscr{T})=\mathscr{T}, \quad \mathscr{T} \geq \mathscr{T}_{00}$, and $n_{00} / 2$ and $\mathscr{T}_{00} / 2$ are lower bounds.

regularization of $a_{0}$

We define the regularization, $a_{00}$, of $a_{0}$ to be the diagonal matrix obtained by the replacements:

$$
n \mapsto \tilde{n}, \quad \mathbf{v} \mapsto \mathbf{v}, \quad \mathscr{T} \mapsto \tilde{T}
$$

\section{regularization of $L$}

A similar statement applies to the regularization $L_{0}$ of $L$. We have: $n \mapsto \tilde{n}, \quad \mathbf{v} \mapsto \mathbf{v}, \quad \mathscr{T} \mapsto \tilde{\mathscr{T}}$.

regularization of $a_{j}$ and $b$

For the regularization $a_{j 0}$ of $a_{j}$, the quotients in the first and final diagonal positions are regularized, and for the regularization $b_{0}$ of $b$, the first two elements in the final row are regularized. Specifically,

$$
\begin{aligned}
& \frac{\mathscr{T} v_{j}}{n} \mapsto \frac{\mathscr{T} v_{j}}{\tilde{n}}, \quad \frac{3 n v_{j}}{2 \mathscr{T}} \mapsto \frac{3 n v_{j}}{2 \tilde{T}}, \\
& \frac{3\left(1-\frac{\mathscr{T} *}{\mathscr{T}}\right)}{2 \tau_{w}} \mapsto \frac{3\left(1-\frac{\mathscr{T} *}{\tilde{T}}\right)}{2 \tau_{w}}, \quad c \frac{n \mathbf{v}}{\mathscr{T}} \mapsto c \frac{n \mathbf{v}}{\tilde{T}} .
\end{aligned}
$$

We are interested then in the regularized system,

$$
\begin{aligned}
& a_{00}(\mathbf{u}) \mathbf{u}_{t}-L_{0}(\mathbf{u}) \mathbf{u}+\left[\sum_{j=1}^{d} a_{j 0}(\mathbf{u}) \frac{\partial \mathbf{u}}{\partial x_{j}}+b_{0}(\mathbf{u}) \mathbf{u}\right] \\
& \quad=0
\end{aligned}
$$

\section{A Framework for Analysis}

We introduce the classical Bessel potential space $H^{s}\left(R^{d} ; R^{k}\right)$ [12]. It can be characterized, via the isometric Fourier transform $\mathscr{F}$, as the linear space of functions $v$ with norm,

$$
\|v\|_{H^{s}}^{2}=\int_{R^{d}}\left(1+|x|^{2}\right)^{s}|\tilde{F} v(x)|^{2} \quad d x .
$$

It follows from the definition that the diagonal operator $S=I(I-\Delta)^{s / 2}$ induces an isometry of $H^{s}\left(R^{d} ; R^{k}\right)$ onto $L_{2}\left(R^{d} ; R^{k}\right)$. Here, $\Delta$ denotes the Laplacian.

We may now define:

$$
X=L_{2}\left(R^{d} ; R^{d+2}\right), \quad Y=H^{s}\left(R^{d} ; R^{d+2}\right) .
$$

Another type of space required for our analysis is the class of uniformly local spaces [13,8: p. 252]. Since these spaces may not be familiar, we recall their definition here.

Definition 3.1 For $\mathscr{H}$ a fixed Hilbert space (including the special case of Euclidean space), let $L_{2, u}\left(R^{d} ; \mathscr{H}\right)$ denote the set of all (equivalence classes of) $\mathscr{H}$ - valued strongly measurable functions $u$ such that

$$
\|u\|=\sup _{x \in R^{d}}\left(\int_{|y-x|<1}|u(y)|^{2} d y\right)^{1 / 2}<\infty .
$$

This space is called a uniformly local $L_{2}$ space. For each integer $s \geq 0$, denote by $H_{u \ell}^{s}$ the set of $u \in L_{2, u \ell}$ such that the distribution derivatives $D^{\alpha} u$ of order $|\alpha| \leq s$ are in $L_{2, u \ell}$. The norm in $H_{u \ell}^{s}$ is given by

$$
\|u\|_{H_{u \ell}^{s}}=\sup _{|\alpha| \leq s}\left\|D^{\alpha} u\right\|_{L_{2, u}} .
$$

Interpolation space theory may be used to extend the definition to non-integral $s$.

We state two essential properties of the uniformly local $H^{s}$ spaces which will be used in the course of our analysis. 


\section{inclusion relations}

If $s>d / 2+k, k$ a nonnegative integer, and $m \geq 1$ is an integer, then

$$
H^{s}\left(R^{d} ; R^{m}\right) \subset H_{u \ell}^{s}\left(R^{d} ; R^{m}\right) \subset C_{b}^{k}\left(R^{d} ; R^{m}\right),
$$

where the inclusions are continuous. Here, the subscript $b$ indicates that derivatives through order $k$ are bounded.

\section{multiplier relations}

If $s>d / 2$, then multiplication induces continuous bilinear maps:

$$
H^{\tau+\sigma}\left(R^{d} ; R^{1}\right) \times H_{u \ell}^{s-\sigma}\left(R^{d} ; R^{m}\right) \mapsto H^{\tau}\left(R^{d} ; R^{m}\right),
$$

for $0 \leq \sigma \leq s, 0 \leq \tau \leq s-\sigma$.

The regularized matrix $a_{00}$ contains entries obtained by taking reciprocals, which are not, in general, $H^{s}$ functions. We have introduced the uniformly local spaces precisely to cover this situation. The regularized reciprocals of $H^{s}$ functions are in $H_{u \ell}^{s}$, and hence are (invariant) multipliers. This pertains as well to the regularizations of $L$ and $a_{j}$.

Now for the regularized problem, we will use the notation $A, \tilde{A}$ of "The General Initial-Value Problem" section, so that $\tilde{A}=A_{0}^{-1} A$. We review some basic facts about semigroup generation for the operator $A[8, \mathrm{pp}$. 231-233]

Lemma 3.1 Let $\mathbf{w} \in Y, s>d / 2+1$. Let

$$
E(\mathbf{w})=\left[\sum_{j=1}^{d} a_{j 0}(\mathbf{w}) \frac{\partial}{\partial x_{j}}+b_{0}(\mathbf{w})\right]
$$

where $a_{j}, b$ are defined in Eq. (1.16) and $a_{j 0}, b_{0}$ are the regularizations. Then the following hold.

1. $E=E(\mathbf{w})$ may be identified with the closed linear operator, defined by the formal adjoint relation,

$$
\left(\mathbf{u}, E^{*} \psi\right)_{X}=(\mathbf{v}, \psi)_{X}, \quad \forall \psi \in C_{0}^{\infty}\left(R^{d}\right),
$$

where $\mathbf{v}=E \mathbf{u}, \quad \mathbf{u} \in \mathscr{D}(E)$.

2. The relation,

$$
(E \psi, \psi)_{X}=\left(\frac{1}{2}\left(b_{0}+b_{0}^{*}-\sum_{j=1}^{d} \frac{\partial a_{j 0}}{\partial x_{j}}\right) \psi, \psi\right)_{X}
$$

holds. In particular, the energy inequality,

$$
(E \mathbf{u}, \mathbf{u})_{X} \geq-\omega_{0}(\mathbf{u}, \mathbf{u})_{X}
$$

holds for all $\mathbf{u} \in \mathscr{D}(E)$, where

$$
\omega_{0}=\frac{1}{2} \sum_{j=1}^{d}\left\|a_{j 0}\right\|_{C^{1}}+\left\|b_{0}\right\|_{C} .
$$

\section{3. $E \in G\left(X, 1, \omega_{0}\right)$.}

Proof We give only a sketch, referring the reader to the above reference. Property (1) is due to Friedrichs [14,15]. Property (2) is a direct calculation, and uses the Hermitian symmetry of the matrices $a_{j 0}$. The energy inequality is an immediate consequence, and in turn, implies the generation property for $-E$, via the Hille-Yosida theorem.

We now discuss the complete spatial operator given by Eq. (3.3).

Lemma 3.2 Let $\mathbf{w} \in Y, s>d / 2+1$. The sum,

$$
A(\mathbf{w})=L_{0}(\mathbf{w})+\left[\sum_{j=1}^{d} a_{j 0}(\mathbf{w}) \frac{\partial}{\partial x_{j}}+b_{0}(\mathbf{w})\right],
$$

is in $G(X, 1, \omega)$. Here, $\omega$ is proportional to the cube of the radius $r$ in $Y$ of the ball from which the coefficients of $L_{0}$, $a_{j 0}, b_{0}$ are taken.

Proof This is straightforward after an initial observation. One observes that the energy inequality of the previous lemma holds for the augmented operator, on a pre-domain of compact support functions. We illustrate this by examining the action of the final component of $L_{0}(\mathbf{w})$. Let $\psi \in C_{0}^{\infty}\left(R^{d} ; R\right)$, and consider the $L_{2}$ inner product,

$$
(-\nabla \cdot(\tilde{n} \nabla \psi), \psi / \tilde{\mathscr{T}})_{L_{2}},
$$

where $n, \mathscr{T}$ are components of $\mathbf{w}$. After integration by parts and an application of the product rule to $\psi / \tilde{\mathscr{T}}$, we obtain

$$
\begin{aligned}
(-\nabla \cdot(\tilde{n} \nabla \psi), \psi / \tilde{\mathscr{T}})_{L_{2}}= & ((\tilde{n} / \tilde{\mathscr{T}}) \nabla \psi, \nabla \psi)_{L_{2}} \\
& -\left(\left(\tilde{n} / \tilde{\mathscr{T}}^{2}\right) \nabla \psi, \psi \nabla \tilde{\mathscr{T}}\right)_{L_{2}} .
\end{aligned}
$$

The second term on the right hand side is estimated via:

$$
\begin{aligned}
\left(\left(\tilde{n} / \tilde{\mathscr{T}}^{2}\right) \nabla \psi, \psi \nabla \tilde{\mathscr{T}}\right)_{L_{2}} \geq & -\delta((\tilde{n} / \tilde{\mathscr{T}}) \nabla \psi, \nabla \psi)_{L_{2}} \\
& -C_{\delta}(\psi, \psi)_{L_{2}},
\end{aligned}
$$

where $\delta$ can be made arbitrarily small. Here, $C_{\delta}$ depends upon $r$ as stated in the lemma. One forms the sum, $A(\mathbf{w})$, deduces its action on $C_{0}^{\infty}\left(R^{d} ; R^{d+2}\right)$ by the preceding lemma, and then takes the closure of this operator.

In the following definition, we shall create the inductive framework required for the application of Proposition 2.4.

Definition 3.2 Suppose that $\Delta t=T / N$, and $\mathbf{u}_{1}^{N}, \ldots, \mathbf{u}_{k-1}^{N}$ are given as solutions of the semidiscretization (2.3) of the regularized problem (3.3) for $i=1, \ldots, k-1$, where $k \leq N$ is arbitrary. We suppose also that $\mathbf{u}_{k}^{N}$ is arbitrary in $Y$. Thus, we have

$$
A\left(t_{i}, \mathbf{u}_{i}^{N}\right):=A\left(t_{i}\right)=L_{0}\left(\mathbf{u}_{i}^{N}\right)+\left[\sum_{j=1}^{d} a_{j 0}\left(\mathbf{u}_{i}^{N}\right) \frac{\partial}{\partial x_{j}}+b_{0}\left(\mathbf{u}_{i}^{N}\right)\right] .
$$

We define a new norm at the points $t_{i}=i \Delta t$ :

$$
\|\mathbf{f}\|_{t}=\left\|a_{00}(t) \mathbf{f}\right\|_{X}, \quad t=t_{i},
$$


where $a_{00}(t)$ is given by

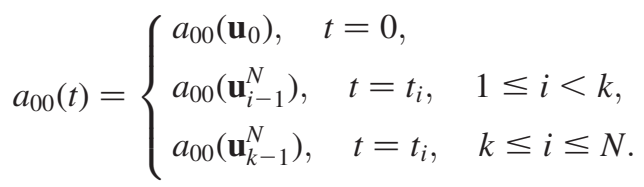

Lemma 3.3 Suppose that, in the previous definition, there is a constant $c^{\prime}$, such that

$$
\left\|\mathbf{u}_{i}^{N}-\mathbf{u}_{i-1}^{N}\right\|_{C} \leq c^{\prime} \Delta t, \quad i=1, \ldots, k-1 .
$$

Then the norms as defined in Eqs. (3.8) and (3.9) satisfy the condition (2.12) at the points $t_{i}$.

Proof We have:

$$
\frac{\|\mathbf{f}\|_{t}}{\|\mathbf{f}\|_{s}}=\frac{\left\|a_{00}(t) \mathbf{f}\right\|_{X}}{\left\|a_{00}(s) \mathbf{f}\right\|_{X}} \leq\left\|a_{00}(t) a_{00}^{-1}(s)\right\| .
$$

By interchanging the roles of $s$ and $t$ we may assume that $t \geq s$, and without loss of generality we assume $t>s \geq 0$. The individual main-diagonal entries of the diagonal matrix,

$$
a_{00}(t) a_{00}^{-1}(s),
$$

are quotients, or a simple product of quotients. These basic quotients are of the form,

$$
\frac{\tilde{n}(n(t))}{\tilde{n}(n(s))}, \quad \frac{\tilde{\mathscr{T}}(\mathscr{T}(t))}{\tilde{\mathscr{T}}(\mathscr{T}(s))}
$$

or their reciprocals. We use a simple algebraic relation to estimate these quotients:

$$
\left|\frac{\alpha}{\beta}\right| \leq(1+C|\alpha-\beta|),
$$

where $C \geq 1 / \beta$. It will be enough to consider certain case distinctions. These are made so that the term $|\alpha-\beta|$ is easily computable.

Case I $t_{i}=s<t=t_{i+1}, 1 \leq i \leq N-1$

Note that the case $i=0$ is trivial. For concreteness, we consider the quotient,

$$
\frac{\tilde{n}(n(t)}{\tilde{n}(n(s)} .
$$

Its simple estimation is bounded above via (3.12) and the definitions of $\tilde{n}, a_{00}$ by:

$$
\left(1+C_{1}\left\|n_{i}-n_{i-1}\right\|_{C}\right), \quad 1 \leq i \leq N-1,
$$

for some constant $C_{1}$. This makes use of the Sobolev embedding theorem and the smoothness of the function $\zeta$. A similar estimate is derivable for the reciprocal, and for the expressions in $\mathscr{T}$. We have derived an upper bound of at most

$$
\left(1+C_{0} \Delta t\right)^{2}
$$

for the individual entries of $a_{00}\left(t_{i+1}\right) a_{00}^{-1}\left(t_{i}\right)$, upon use of the hypothesis (3.10). Elementary properties of the norm give the same bound on the operator norm of $a_{00}\left(t_{i+1}\right) a_{00}^{-1}\left(t_{i}\right)$. In particular, we have Eq. (2.12) in this case upon use of the bounding exponential function.

Case II $t=t_{i}>s=t_{j} \geq 0$

In this case, we compare successively adjacent intervals in the obvious way. This yields Eq. (2.12) in the general case with $c_{0}=2 C_{0}$.

One final important result is required, pertaining to Proposition 2.7. It is fundamental.

\section{The Commutator Estimate Linking $X$ And $Y$}

We quote here the fundamental commutator result of Kato [7, Appendix], as adapted to our present context. We retain the meaning of $a_{00}(t)$ as defined in Eq. (3.9).

LeMma 3.4 For a function $\mathbf{v} \in H^{s+1}\left(R^{d} ; R^{k}\right)$, and an operator of the form,

$$
\begin{aligned}
& \tilde{A}(t, v)=a_{00}^{-1}(t) \\
& \left\{L_{0}(\mathbf{v})+\left[\sum_{j=1}^{d} a_{j 0}(\mathbf{v}) \frac{\partial}{\partial x_{i}}+b_{0}(\mathbf{v})\right]\right\},
\end{aligned}
$$

we have

$$
\begin{aligned}
& S \tilde{A}(t, \mathbf{v}) S^{-1}=\tilde{A}(t, \mathbf{v}) \\
& +\left[-\left[S, a_{00}^{-1} D_{0}\right] \Lambda^{1-(s-1)}\left(\Lambda^{-2} \nabla \cdot(\tilde{n} \nabla)\right)\right. \\
& \left.+\sum_{j=1}^{d}\left[S, a_{00}^{-1} a_{j 0}\right] \Lambda^{1-s}\left(\frac{\partial}{\partial x_{j}}\right) \Lambda^{-1}+\left[S, a_{00}^{-1} b_{0}\right] \Lambda^{1-s} \Lambda^{-1}\right],
\end{aligned}
$$

where $D_{0}=\operatorname{diag}(0, \mathbf{0}, 1 / \tilde{\mathscr{T}}), \Lambda=(I-\Delta)^{1 / 2},[\cdot, \cdot]$ denotes the commutator, and $S=I_{k} \Lambda^{s}$; here $I_{k}$ is the identity matrix of order $k$. In particular, in the notation of Proposition 2.7, we have

$$
\begin{aligned}
& \tilde{B}=\left[-\left[S, a_{00}^{-1} D_{0}\right] \Lambda^{1-(s-1)} \Lambda^{-2}(\nabla \cdot(\tilde{n} \nabla))\right. \\
& \left.+\sum_{j=1}^{d}\left[S, a_{00}^{-1} a_{j 0}\right] \Lambda^{1-s}\left(\frac{\partial}{\partial x_{j}}\right) \Lambda^{-1}+\left[S, a_{00}^{-1} b_{0}\right] \Lambda^{1-s} \Lambda^{-1}\right] .
\end{aligned}
$$


If $s>d / 2+1$, then $B$ is a bounded operator on $L_{2}\left(R^{d} ; R^{k}\right)$ with bound:

$$
\begin{aligned}
& \|\tilde{B}\| \leq C\left(\left\|\operatorname{grad} a_{00}^{-1} D_{0}\right\|_{H^{s}}+\sum_{j=1}^{d}\left\|\operatorname{grad} a_{00}^{-1} a_{j 0}\right\|_{H^{s-1}}\right. \\
& \left.\quad+\left\|\operatorname{grad} a_{00}^{-1} b_{0}\right\|_{H^{s-1}}\right)
\end{aligned}
$$

Note that we have used the fact that $\Lambda^{-2}(\nabla \cdot(\tilde{n} \nabla))$ can be extended to be a bounded linear operator on $L_{2}\left(R^{d} ; R^{k}\right)$. This is where we need the increase (by one) of $s$, in the hypothesis on $\mathbf{v}$. Thus, we now assume $s>d / 2+2$.

\section{Stability on $X$ and $Y$}

We suppose that we are in the inductive situation of Definition 2.2. We shall now deduce stability for the regularized problem.

Proposition 3.1 In the context of Definition 2.2, we have stability on $X$ and $Y$, provided Eq. (3.10) holds. In particular, Eq. (3.10) holds, with $c^{\prime}$ a sixth degree polynomial of $r$, if a fixed $r$ is chosen independently of $k$. Here, $r$ is the radius of the admissible $\bar{W}_{0}$ in $Y$ on which $Q$ acts. More precisely, the constants have values $M_{t}=1$, $\omega_{t}=C r^{3}$ on any $X_{t}$, so that, on $X, M$ is proportional to $r^{2}$ and $\omega=C r^{3}$. On $Y$, we have $M_{1}$ proportional to $r^{2}$ and $\omega_{1}$ a quintic function of $r$. The stability constant $c$ is a constant multiple of $c^{\prime}$ for both $X$ and $Y$.

Proof The characterization of $c^{\prime}$ as a sixth degree polynomial is a consequence of the analysis of Lemma 3.5 to follow: a direct estimate of the semidiscrete equation gives a product of a cubic polynomial with $r$, as an estimate for the differences $\left\|\mathbf{u}_{j}^{N}-\mathbf{u}_{j-1}^{N}\right\|_{C} / \Delta t$. The remaining statements follow from Propositions 2.6 and 2.7, taken together with Lemma 3.3 and 3.4. One begins with $\|\cdot\|_{X}$, estimates with respect to $\|\cdot\|_{0}$, and then applies the above cited results. Note that $\|\tilde{B}(t)\|_{X}$ is a constant multiple of $r^{5}$.

\section{The Lipschitz Properties of $\tilde{A}(\mathbf{u})$}

Recall the definitions of $E$ and $A$ given in Eqs. (3.4) and (3.7). Again, we define $\tilde{A}=a_{00}^{-1} A$. A precise statement of the latter is given in the following lemma.

LEMmA 3.5 The mapping $\mathbf{w} \mapsto E(\mathbf{w}) \in B\left(H^{s}, H^{\tau}\right)$ is Lipschitz continuous in the norm topology for $0 \leq \tau \leq$ $s-1$ for $s>d / 2+1$ :

$$
\left\|E(\mathbf{w})-E\left(\mathbf{w}^{\prime}\right)\right\|_{H^{s}, H^{\tau}} \leq C\left\|\mathbf{w}-\mathbf{w}^{\prime}\right\|_{H^{\tau}}, \quad \mathbf{w}, \mathbf{w}^{\prime} \in \bar{W}_{0} .
$$

The constant $C$ is proportional to a cubic function of the radius $r$ of $\bar{W}_{0} \subset Y$. Similarly, if $s>d / 2+2$, the mapping $\mathbf{w} \mapsto A(\mathbf{w}) \in B\left(H^{s}, H^{\tau}\right)$ is Lipschitz continuous in the norm topology for $0 \leq \tau \leq s-2$ :

$$
\left\|A(\mathbf{w})-A\left(\mathbf{w}^{\prime}\right)\right\|_{H^{s}, H^{\tau}} \leq C\left\|\mathbf{w}-\mathbf{w}^{\prime}\right\|_{H^{\tau}}, \quad \mathbf{w}, \mathbf{w}^{\prime} \in \bar{W}_{0} .
$$

The dependence on $C$ is cubic in $r$. Finally, for fixed $\mathbf{v} \in H^{s}$, the mapping $\mathbf{w} \mapsto \tilde{A}_{\mathbf{v}}(\mathbf{w}) \in B\left(H^{s}, H^{\tau}\right)$ is Lipschitz continuous in the norm topology for $0 \leq \tau \leq$ $s-2$. Here, $\tilde{A}_{\mathbf{v}}(\mathbf{w})=a_{00}^{-1}(\mathbf{v}) A(\mathbf{w})$. In this case, the dependence is quintic in $r$.

Proof We first note the inequalities,

$$
\begin{gathered}
\left\|a_{j 0}(\mathbf{w})-a_{j 0}\left(\mathbf{w}^{\prime}\right)\right\|_{H^{\tau}} \leq c_{1}\left\|\mathbf{w}-\mathbf{w}^{\prime}\right\|_{H^{\tau}} \\
\left\|b_{0}(\mathbf{w})-b_{0}\left(\mathbf{w}^{\prime}\right)\right\|_{H^{\tau}} \leq c_{2}\left\|\mathbf{w}-\mathbf{w}^{\prime}\right\|_{H^{\tau}} .
\end{gathered}
$$

These inequalities use the definitions of the matrices $a_{j 0}, j=1, \ldots, d, b_{0}$, as well as the assumed properties of the mapping $\Phi$. The constants $c_{1}, c_{2}$ depend quadratically upon $r$. Now, since $H^{s-1}$ functions are multipliers on $H^{\tau}$, we have:

$$
\begin{aligned}
& \left\|E(\mathbf{w}) \mathbf{v}-E\left(\mathbf{w}^{\prime}\right) \mathbf{v}\right\|_{H^{s}, H^{\tau}} \\
& \leq c\left(\sum_{j=1}^{d}\left\|a_{j 0}(\mathbf{w})-a_{j 0}\left(\mathbf{w}^{\prime}\right)\right\|_{H^{\tau}}\left\|\frac{\partial \mathbf{v}}{\partial x_{j}}\right\|_{H^{s-1}}\right. \\
& \left.+\left\|b_{0}(\mathbf{w})-b_{0}\left(\mathbf{w}^{\prime}\right)\right\|_{H^{\tau}}\|\mathbf{v}\|_{H^{s-1}}\right) \\
& \leq C^{\prime}\left\|\mathbf{w}-\mathbf{w}^{\prime}\right\|_{H^{\tau}}\|\mathbf{v}\|_{H^{s}} .
\end{aligned}
$$

This gives the statement of the lemma regarding $E$. To obtain the statement regarding $A$, we examine $L_{0}$. If $v_{d+2}$ denotes the last component of $\mathbf{v}$, we have:

$$
\begin{aligned}
& \left\|L_{0}(\mathbf{w}) \mathbf{v}-L_{0}\left(\mathbf{w}^{\prime}\right) \mathbf{v}\right\|_{H^{s}, H^{\tau}} \\
& \quad \leq c\left\{\left\|D_{0}(\mathbf{w})-D_{0}\left(\mathbf{w}^{\prime}\right)\right\|_{H^{\tau}}\left\|\nabla \cdot\left(n \nabla v_{d+2}\right)\right\|_{H^{s-2}}\right. \\
& \left.\quad+\left\|D_{0}\left(\mathbf{w}^{\prime}\right)\left(\nabla\left(n-n^{\prime}\right) \cdot \nabla v_{d+2}+\left(n-n^{\prime}\right) \Delta v_{d}\right)\right\|_{H^{\tau}}\right\} \\
& \quad \leq C^{\prime}\left\|\mathbf{w}-\mathbf{w}^{\prime}\right\|_{H^{\tau}},
\end{aligned}
$$

provided $s>d / 2+2$. Here, we have made use of the multiplier relations. The same observations concerning the cubic growth in $r$ apply. Finally, in order to analyze $\tilde{A}$, we note that the explicit operator $a_{00}^{-1}$ is a multiplier on each $H^{\tau}$ space; the estimate contributes an additional factor proportional to $r^{2}$.

\section{The Fixed Point Theorem for the Bløtekjær System}

As in the discussion of "The general initial-value problem" section, we define $\bar{\omega}$ to be the maximum of the constants $\omega$ and $\omega_{1}$, derived from the classes $G(X, M, \omega)$ and $G\left(Y, M, \omega_{1}\right)$. We note that $\bar{\omega}$ depends polynomially on $r$, the radius of the admissible set $\bar{W}_{0}$. 
Since $M$ and $c$ do not change with $X$ and $Y$, we retain this notation.

Local Assumption on $\left\|\boldsymbol{u}_{0}\right\|$ and $T$ If $M(r)$ is the stability constant of Definition 2.2, and $c=c(r)$ is given in this definition, both analyzed in Proposition 3.1, we require:

$$
\left\|\mathbf{u}_{0}\right\|_{Y}<\frac{r}{M(r)} e^{-(1+\bar{\omega}(r)+c(r)) T}:=H(r, T)
$$

Equation (3.14) is the general inequality, which must be satisfied by $\left\|\mathbf{u}_{0}\right\|_{Y}, T$, and the radius $r$ of the admissible ball in $Y$. This is quite general: $T$ may be given arbitrarily.

We now define numbers $\delta$ and $\rho$ which allow us to connect Eq. (3.14) with the theoretical analysis of Propositions 2.3 and 2.4. Set $\gamma=1+\bar{\omega}+c$, and select $\rho$ satisfying

$$
M(r)\left\|\mathbf{u}_{0}\right\|_{Y} e^{(1+1 / \rho) \gamma(r) T}<r,
$$

which is possible by Eq. (3.14). Define:

$$
\delta=r e^{-(1+1 / \rho) \gamma(r) T} /\left(M(r)\left\|\mathbf{u}_{0}\right\|_{Y}\right)-1 .
$$

It is immediate that

$$
(1+\delta) M(r) e^{(1+1 / \rho) \gamma(r) T}\left\|\mathbf{u}_{0}\right\|_{Y}=r
$$

We further define:

$$
\sigma=(1+\delta) M(r) e^{(1+1 / \rho) \gamma(r) T} .
$$

These definitions then describe the framework investigated in Propositions 2.3 and 2.4. In particular,

$$
\sigma\left\|\mathbf{u}_{0}\right\|_{Y}=r .
$$

Assumption on $\Phi$ Prior to the statement of the theorem, we formally state the smoothing assumption regarding the mapping $\Phi$ of Eq. (1.17).

Remark 3.1 It is assumed that the affine mapping $\Phi$ depends smoothly upon the first component of $\mathbf{u}$ in the following sense: Given $u_{1} \in H^{\tau}\left(R^{d} ; R^{1}\right), 0 \leq \tau \leq s$, the solution $\phi$ of Eq. (1.17) is in $H^{\tau+2}\left(R^{d}\right)$, and the norm of $\phi$ in this space is affinely dominated by the $H^{\tau}$ norm of $u_{1}$, with constants independent of $\tau$ and $u_{1}$.

We then have the following theorem, which follows in a direct manner from the framework we have developed.

TheOREM 3.1 If Ineq. (3.14) holds, and $N$ is sufficiently large, then the mapping $Q$, with Lipschitz constant $C_{Q}$ given by Eq. (2.11), is a strict contraction on $\bar{W}_{0}$. In this case, $Q$ has a unique fixed point, denoted $\mathbf{u}_{k}^{N}$. The implicit relation (1.17) is satisfied.

\section{ANALYSIS ON THE SPACE-TIME DOMAIN}

This part of the paper is more routine than the earlier parts, and we shall summarize the principal elements of the convergence theory for readers unacquainted with this approach. The author has given greater detail in Ref. [16], and supporting detail in Refs. [8,17].

The idea is to define step function or piecewise linear sequences in time which make use of the semidiscrete spatial solutions. Weak and local strong compactness allow one to obtain a unique limit. This limit is first shown in the theory to be a weak solution, and then a strong solution in certain regularity classes. The strong solution is not only unique; it is invariant under $\kappa_{0} \rightarrow 0$.

We begin by defining the relevant sequences which make use of the semidiscrete solutions.

Definition 4.1 For $\Delta t=T / N, t_{k}=k \Delta t$, and $0 \leq t \leq$ $T$, set

$$
\theta_{k}^{N}(t)=\left\{\begin{array}{lc}
1, & t_{k-1} \leq t<t_{k} \quad k=1, \ldots, N \\
0, & \text { otherwise }
\end{array}\right.
$$

Then for $x \in R^{d}$, define:

$$
\begin{gathered}
\mathbf{u}_{P L}^{N}(x, t)=\mathbf{u}_{k}^{N}(x)+\frac{t-t_{k}}{\Delta t}\left(\mathbf{u}_{k}^{N}(x)-\mathbf{u}_{k-1}^{N}(x)\right), \\
t_{k-1} \leq t<t_{k}, \\
k=1, \ldots, N \\
\mathbf{u}_{S}^{N}(x, t)=\sum_{k=1}^{N} \mathbf{u}_{k}^{N}(x) \theta_{k}^{N}(t), \\
a_{j 0}^{N}(x, t)=a_{j 0}\left(\mathbf{u}_{S}^{N}(x, t)\right), \\
b_{0}^{N}(x, t)=b_{0}\left(\mathbf{u}_{S}^{N}(x, t)\right) .
\end{gathered}
$$

We also require function space notation:

$$
\mathscr{Y}=W_{\infty}^{1}\left((0, T) ; H^{s-2}\left(R^{d} ; R^{d+2}\right)\right) \cap L_{\infty}((0, T) ; Y) .
$$

Lemma 4.1 The sequence $\left\{\mathbf{u}_{S}^{N}\right\}$ is bounded in norm in $L_{\infty}((0, T) ; Y)$. The sequence $\left\{\mathbf{u}_{P L}^{N}\right\}$ is bounded in norm in $\mathscr{Y}$.

Proof The boundedness of both sequences in $L_{\infty}((0, T) ; Y)$ reflects the construction of the sequences via the fixed points of the mapping $Q$. To establish the boundedness of

$$
\frac{\partial \mathbf{u}_{P L}^{N}}{\partial t}=\frac{\mathbf{u}_{k}^{N}-\mathbf{u}_{k-1}^{N}}{\Delta t}
$$

in $L_{\infty}\left((0, T) ; H^{s-2}\left(R^{d}\right)\right)$, we directly take the norm in the Eq. (2.3). For the term, $A\left(\mathbf{u}_{k}^{N}\right) \mathbf{u}_{k}^{N}$, we use the $Y$ bound for $\mathbf{u}_{k}^{N}$, together with the uniform estimate on $A\left(\mathbf{u}_{k}^{N}\right)$ from $H^{s}\left(R^{d} ; R^{d+2}\right)$ to $H^{s-2}\left(R^{d} ; R^{d+2}\right)$, which can be deduced from Lemma 3.5. 
Remark 4.1 By the Aubin lemma ([8, p. 197]),

$$
\mathscr{Y} \hookrightarrow{ }^{\text {compactly }} L_{2, \text { loc }}(\mathscr{D}) \text {. }
$$

Here, we have used the notation "loc" to indicate convergence on bounded subsets of $\mathscr{D}=(0, T) \times R^{d}$.

\section{The Weak Solution}

We shall use compactness arguments to obtain a weak solution, $\mathbf{u}$. We have the following.

THEOREM 4.1 There are subsequences, denoted $\mathbf{u}_{P L}^{N_{j}}, \mathbf{u}_{S}^{N_{j}}$, and a function $\mathbf{u} \in \mathscr{Y}$, satisfying the constraint (1.17), such that:

$$
\begin{aligned}
& \mathbf{u}_{P L}^{N_{j}} \rightarrow \mathbf{u} \text { weakly in } L_{2}((0, T) ; Y), \\
& \mathbf{u}_{P L}^{N_{j}}{ }^{*}{ }^{*} \mathbf{u} \text { weak }-{ }^{*} \text { in } W_{\infty}^{1}\left((0, T) ; H^{s-2}\left(R^{d}\right)\right. \\
& \cap L_{\infty}((0, T) ; Y), \\
& \mathbf{u}_{P L}^{N_{j}} \rightarrow \mathbf{u} \text { in } L_{2, \text { loc }}(\mathscr{D}), \\
& \mathbf{u}_{S}^{N_{j}} \rightarrow \mathbf{u} \text { weakly in } L_{2}((0, T) ; Y) \text {, } \\
& \mathbf{u}_{S}^{N_{j}} \rightarrow \mathbf{u} \text { in } L_{2, \operatorname{loc}}(\mathscr{D}), \\
& a_{j 0}^{N_{j}} \rightarrow a(\cdot, \mathbf{u}) \text { in } L_{2, \text { loc }}(\mathscr{D}) \text {, } \\
& b^{N_{j}} \rightarrow b(\cdot, \mathbf{u}) \text { in } L_{2, \mathrm{loc}}(\mathscr{D})
\end{aligned}
$$

The function $\mathbf{u}$ is a weak solution of the Cauchy problem: if $\psi \in C^{\infty}\left([0, T] ; C_{0}^{\infty}\left(R^{d}, R^{d+2}\right)\right)$, and $T^{\prime} \leq T$, then, for $\mathscr{D}_{T^{\prime}}=R^{d} \times\left(0, T^{\prime}\right)$,

$$
\begin{aligned}
& \int_{D_{T^{\prime}}}\left\{\mathbf{u} \psi_{t}-A(\cdot, \mathbf{u}) \mathbf{u} \psi\right\} \mathrm{d} x \mathrm{~d} t+\int_{R^{d} \times\{0\}} \mathbf{u}_{0} \psi \mathrm{d} x \\
& \quad-\int_{R^{d} \times\left\{T^{\prime}\right\}} \mathbf{u} \psi \mathrm{d} x \\
& =0 .
\end{aligned}
$$

Proof The Aubin lemma shows that the limits in Eqs. (4.5) and (4.6), which exist by weak compactness, coincide and lead to Eq. (4.7). In this connection, recall that a compact mapping (injection) maps weakly convergent sequences onto strongly convergent sequences. That the limit in Eq. (4.8) may be taken to be $\mathbf{u}$ follows from Eq. (4.5) and Lemma 4.1; in particular, from the uniform $H^{s-2}$ bound for

$$
\frac{\mathbf{u}_{k}^{N}-\mathbf{u}_{k-1}^{N}}{\Delta t}
$$

(see Ref. [8, Lemma 5.2.6]). This bound also implies that the limits in Eqs. (4.7) and (4.9) coincide. The limits in Eqs. (4.10) and (4.11), and the constraint (1.17), follow from the definitions and the assumed properties of the mapping $\Phi$. Define $\psi_{k}^{N}=1 / \Delta t \int_{t_{k-1}}^{t_{k}} \psi(x, t) \mathrm{d} t, k=1, \ldots, N$, and $\psi^{N}=\sum_{k=1}^{N} \psi_{k}^{N} \theta_{k}^{N}$. We dot multiply Eq. (2.3) by $\psi_{k}^{N}$, sum on $k, k=1, \ldots, N$, and integrate over $R^{d}$ to obtain:

$$
\begin{aligned}
& \sum_{k=1}^{N-1}\left(\mathbf{u}_{k}^{N}, \frac{\psi_{k}^{N}-\psi_{k+1}^{N}}{\Delta t}\right)_{L_{2}} \Delta t+\left(\mathbf{u}_{N}^{N}, \psi_{N}^{N}\right)_{L_{2}}-\left(\mathbf{u}_{0}, \psi_{1}^{N}\right)_{L_{2}} \\
& \quad+\sum_{k=1}^{N}\left(\tilde{A}\left(\mathbf{u}_{k}^{N}\right) \mathbf{u}_{k}^{N}, \psi_{k}^{N}\right)_{L_{2}} \Delta t \\
& \quad=0
\end{aligned}
$$

If one rewrites this expression, it becomes, with $\zeta^{N}=\sum_{k=1}^{N-1}\left(\psi_{k}^{N}-\psi_{k+1}^{N}\right) \theta_{k}^{N} / \Delta t$

$$
\begin{aligned}
& \int_{0}^{(N-1) \Delta t}\left(\mathbf{u}_{S}^{N}, \zeta^{N}\right)_{L_{2}} \mathrm{~d} t+\left(\mathbf{u}_{N}^{N}, \psi_{N}^{N}\right)_{L_{2}}-\left(\mathbf{u}_{0}, \psi_{1}^{N}\right)_{L_{2}} \\
& \quad+\int_{0}^{T}\left(\tilde{A}\left(\mathbf{u}_{S}^{N}\right) \mathbf{u}_{S}^{N}, \psi^{N}\right)_{L_{2}} \mathrm{~d} t \\
& \quad=0
\end{aligned}
$$

By Ref. [8, Lemma 5.2.5], it follows that

$$
\psi^{N} \rightarrow \psi \text { in } L_{2}(\mathscr{D}), \quad \zeta^{N} \rightarrow-\frac{\partial \psi}{\partial t} \text { in } L_{2}(\mathscr{D}) .
$$

We now allow $N=N_{j} \rightarrow \infty$. The terms involving $\tilde{A}$ are analyzed by Lemma 3.5. Further, $\int_{\left(N_{j}-1\right) \Delta t}^{T}\left(\mathbf{u}_{S}^{N_{j}}, \zeta^{N_{j}}\right)_{L_{2}} \mathrm{~d} t \rightarrow 0$, by the pointwise boundedness of the integrated sequences. In order to analyze $\left(\mathbf{u}_{0}, \psi_{1}^{N_{j}}\right)_{L_{2}}$, we use the mean value theorem of integral calculus to deduce that $\psi_{1}^{N_{j}}(x)=\psi(x, t(x))$, for some $0<t(x)<\Delta t$. Uniform continuity then leads to

$$
\left(\mathbf{u}_{0}, \psi_{1}^{N_{j}}\right)_{L_{2}} \rightarrow \int_{R^{d} \times\{0\}} \mathbf{u}_{0} \psi \mathrm{d} x
$$

One may then use the fundamental theorem of calculus in reflexive Banach spaces [18] to deduce that $\left(\mathbf{u}_{N_{j}}^{N_{j}}, \psi_{N_{j}}^{N_{j}}\right)_{L_{2}} \rightarrow \int_{R^{d} \times\{T\}} \mathbf{u} \psi \mathrm{d} x$. To see this, one argues as follows. The intermediate representation,

$$
\left(\mathbf{u}_{N_{j}}^{N_{j}}, \psi\right)_{L_{2}}-\left(\mathbf{u}_{0}, \psi\right)_{L_{2}}=\int_{0}^{T}\left[\left(\mathbf{u}_{P L}^{N_{j}}, \psi\right)_{L_{2}}\right]_{t} \mathrm{~d} t,
$$

and its limit are used to deduce that $\left(\mathbf{u}_{N_{j}}^{N_{j}}, \psi\right)_{L_{2}} \rightarrow$ $\int_{R^{d} \times\{T\}} \mathbf{u} \psi \mathrm{d} x$. Use of the uniform convergence of the $\psi$-averages and the triangle inequality completes the argument. We thus have the limit rendered by Eq. (4.12) for $T=T^{\prime}$. The general case is similar.

\section{Existence and Uniqueness of Strong Solutions}

Before stating the result on strong solutions extending Eq. (4.12), we require a technical estimation lemma, used in the regularity argument for solutions. We shall simply 
quote the lemma, since a slightly generalized version was proved in Ref. [16].

Lemma 4.2 The estimate,

$$
\left\|\mathbf{u}_{k}^{N}\right\|_{H^{s}}^{2} \leq\left\|\mathbf{u}_{0}\right\|_{H^{s}}^{2}+C \sum_{j=1}^{k}\left\|\mathbf{u}_{j}^{N}\right\|_{H^{s}}^{2} \Delta t
$$

holds for some constant $C$ independent of $N$. If

$$
\alpha^{N}(t)=\left\{\sum_{k=1}^{N}\left\|\mathbf{u}_{k}^{N}\right\|_{H^{s}}^{2} \theta_{k}^{N}(t)\right\}, \quad \alpha(t)=\sup _{N}\left\{\alpha^{N}(t)\right\},
$$

then $\alpha \in L_{1}$, and it follows that

$$
\|\mathbf{u}(t)\|_{H^{s}}^{2} \leq\left\|\mathbf{u}_{0}\right\|_{H^{s}}^{2}+C \int_{0}^{t} \alpha(\tau) \mathrm{d} \tau
$$

We have the following.

Corollary 4.1 The solution $\mathbf{u}$ of Eqs. (4.12) and (1.17) is a strong solution. Specifically,

$$
\mathbf{u}_{t} \in C\left([0, T] ; H^{s-2}\left(R^{d}\right)\right),
$$

and the Eq. (3.3) holds in the strong sense described by Eq. (4.15). Moreover,

$$
\mathbf{u} \in C\left([0, T] ; H^{s}\left(R^{d}\right)\right) .
$$

Proof The regularity (4.15) follows from Eq. (4.6) of Theorem 4.1 and the fundamental theorem of calculus in reflexive Banach spaces. This also validates an integration by parts, and hence the strong form of the evolution equation. Note that each of the terms in Eq. (1.15) (more precisely, in the regularized system (3.3)) is in the class (4.15) (see Lemma 3.5). The regularity (4.16) is more subtle and can be deduced from

$$
\mathbf{u} \in L_{\infty}\left((0, T) ; H^{s}\left(R^{d}\right)\right)
$$

established in Theorem 4.1, in a manner similar to that employed in Ref. [19, pp. 44-46], where it is noted that right continuity at zero suffices to establish the continuity on $[0, T]$. The technique to establish right continuity at zero relies on establishing an estimate of the form,

$$
\|\mathbf{u}(t)\|_{H^{s}}^{2} \leq\|\mathbf{u}(0)\|_{H^{s}}^{2}+\int_{0}^{t} f(\tau) \mathrm{d} \tau
$$

where $f$ is an $L_{1}$ function. This is precisely what was done in Lemma 4.2; we identify $f$ with $\alpha$. We may now proceed as in Ref. [19]. This concludes the proof.

Proposition 4.1 The strong solution of Eq. (3.3) described by Corollary 4.1 is unique.
Proof If $\mathbf{u}_{1}$ and $\mathbf{u}_{2}$ are solutions, then one obtains in the standard way,

$$
\begin{aligned}
\frac{1}{2} \frac{\mathrm{d}}{\mathrm{d} t} & \left\|\mathbf{u}_{1}-\mathbf{u}_{2}\right\|_{L_{2}}^{2}+\left(L_{0}\left(\mathbf{u}_{1}\right)\left(\mathbf{u}_{1}-\mathbf{u}_{2}\right), \mathbf{u}_{1}-\mathbf{u}_{2}\right)_{L_{2}} \\
= & -\left(\left(L_{0}\left(\mathbf{u}_{1}\right)-L_{0}\left(\mathbf{u}_{2}\right)\right) \mathbf{u}_{2}, \mathbf{u}_{1}-\mathbf{u}_{2}\right)_{L_{2}}-\left(E _ { 0 } ( \mathbf { u } _ { 1 } ) \left(\mathbf{u}_{1}\right.\right. \\
& \left.\left.-\mathbf{u}_{2}\right), \mathbf{u}_{1}-\mathbf{u}_{2}\right)_{L_{2}}-\left(\left(E_{0}\left(\mathbf{u}_{1}\right)-E_{0}\left(\mathbf{u}_{2}\right)\right) \mathbf{u}_{2}, \mathbf{u}_{1}\right. \\
& \left.-\mathbf{u}_{2}\right)_{L_{2}} .
\end{aligned}
$$

Here, we have used the notation $E_{0}$ to indicate the first order regularized operator of Eq. (3.3). Noting that both terms on the left hand side of this equation are nonnegative, we have, via Lemma 3.5, the inequality,

$$
\left\|\mathbf{u}_{1}-\mathbf{u}_{2}\right\|_{L_{2}}^{2}(t) \leq C \int_{0}^{t}\left\|\mathbf{u}_{1}-\mathbf{u}_{2}\right\|_{L_{2}}^{2}(\tau) \mathrm{d} \tau
$$

for $0 \leq t \leq T$. Here, $C$ is a positive constant depending only on the smooth norms of $\mathbf{u}_{1}, \mathbf{u}_{2}$. Use of the Gronwall inequality concludes the proof.

\section{Stability Under the Limit of Vanishing Heat Flux}

An important feature of the semigroup-based theory which we have presented in this paper is its ability to permit the passage to the case of the strict gas dynamics limit for the charged fluid; i.e. the passage under the limit $\kappa_{0} \rightarrow 0$. Results of this type were obtained in Refs. [16,17] for incompressible charged fluids, where the relevant parameter is the kinematic viscosity. It was noted in these references that Kato developed his theory to cover a range of applications. The only distinction required when $\kappa_{0}=0$ is discussed in Ref. [7 p. 55] for the semigroup generation on $L_{2}$ (though the application is different, the procedure is the same). In fact, there is only one modification: the domain of $A \mapsto E$ is larger, with regularity index decreased by one. The rigorous argument appears in the proof of Lemma 3.1. However, this has no impact upon the invariance results and fixed point arguments for $Q$, nor upon the arguments in the spacetime domain.

The following result is a natural consequence of our arguments.

Proposition 4.2 There is a strong solution, in the regularity classes defined by Eqs. (4.15) and (4.16), of the regularized system $(3.3) /(1.17)$, with $\kappa_{0}=0$. Moreover, the solution interval is stable under the "singular" limit $\kappa_{0} \rightarrow 0$. More precisely, if $\mathbf{u}_{1}, \mathbf{u}_{2}$, are solutions of Eq. (3.3) for values $\kappa_{0}{ }^{\prime} \geq \kappa_{0}{ }^{\prime \prime} \geq 0$, then there is a constant $C$ such that

$$
\left\|\mathbf{u}_{1}-\mathbf{u}_{2}\right\|_{C\left([0, T] ; L_{2}\left(R^{d}\right)\right)} \leq C\left(\kappa_{0}^{\prime}-\kappa_{0}^{\prime \prime}\right) .
$$

The terminal time $T$ is independent of $\kappa_{0}$. 
Proof We use the notation, $L_{0}{ }^{\prime}, L_{0}{ }^{\prime \prime}$, to distinguish the constants $\kappa_{0}{ }^{\prime}, \kappa_{0}{ }^{\prime \prime}$. A generalization of the identity of the previous proposition yields:

$$
\begin{aligned}
\frac{1}{2} \frac{\mathrm{d}}{\mathrm{d} t} & \left\|\mathbf{u}_{1}-\mathbf{u}_{2}\right\|_{L_{2}}^{2}+\left(L_{0}{ }^{\prime}\left(\mathbf{u}_{1}\right)\left(\mathbf{u}_{1}-\mathbf{u}_{2}\right), \mathbf{u}_{1}-\mathbf{u}_{2}\right)_{L_{2}} \\
= & -\left(\left(L_{0}^{\prime}\left(\mathbf{u}_{1}\right)-L_{0}^{\prime \prime}\left(\mathbf{u}_{1}\right)\right) \mathbf{u}_{2}, \mathbf{u}_{1}-\mathbf{u}_{2}\right)_{L_{2}}-\left(\left(L_{0}{ }^{\prime \prime}\left(\mathbf{u}_{1}\right)\right.\right. \\
& \left.-\left(L_{0}^{\prime \prime}\left(\mathbf{u}_{2}\right)\right) \mathbf{u}_{2}, \mathbf{u}_{1}-\mathbf{u}_{2}\right)_{L_{2}}-\left(E_{0}\left(\mathbf{u}_{1}\right)\left(\mathbf{u}_{1}-\mathbf{u}_{2}\right), \mathbf{u}_{1}\right. \\
& \left.-\mathbf{u}_{2}\right)_{L_{2}}-\left(\left(E_{0}\left(\mathbf{u}_{1}\right)-E_{0}\left(\mathbf{u}_{2}\right)\right) \mathbf{u}_{2}, \mathbf{u}_{1}-\mathbf{u}_{2}\right)_{L_{2}} .
\end{aligned}
$$

The use of the regularity classes and previous estimates yields the inequality, for $0 \leq t \leq T$,

$$
\left\|\mathbf{u}_{1}-\mathbf{u}_{2}\right\|_{L_{2}}^{2}(t) \leq C\left\{\left(\kappa_{0}^{\prime}-\kappa_{0}^{\prime \prime}\right)+\int_{0}^{t}\left\|\mathbf{u}_{1}-\mathbf{u}_{2}\right\|_{L_{2}}^{2}(\tau) \mathrm{d} \tau\right\},
$$

for some constant $C$. Use of the Gronwall inequality concludes the proof.

\section{Summation}

We can now complete the analysis of the well-posedness of the model by removing the presence of the regularization. We do require, however, the specification of threshold parameters. More precisely, we assume that Eq. (3.2) is satisfied, which implicitly requires the uniform boundedness of $n_{0}, \mathscr{T}_{0}$ away from zero, and also sets lower bounds for the regularized problem.

THEOREM 4.2 If Eq. (3.2) is satisfied, and $\mathbf{u}$ is the unique solution on $[0, T]$ satisfying Corollary 4.1 and Proposition 4.1 , then there is a maximum subinterval $\left[0, T^{\prime}\right]$ of $[0, T]$ such that $n \geq n_{00}, \mathscr{T} \geq \mathscr{T}_{00}$ on this subinterval and such that the regularized problem (3.3) is identical to the given system (1.15).

Proof The proof is immediate because of the regularity class (4.15) and the Sobolev embedding theorem, which together guarantee that $\mathbf{u}(x, t)$ is bounded and uniformly continuous as a function of $x, t$. Thus, given a unique strong solution $\mathbf{u}$ of Eq. (3.3) on $[0, T]$, one defines:

$$
\begin{aligned}
T^{\prime} & =\max \left\{t^{\prime}: u_{1}(\cdot, t) \geq n_{00}, 0 \leq t \leq t^{\prime}, u_{d+2}(\cdot, t)\right. \\
& \left.\geq \mathscr{T}_{00}, 0 \leq t \leq t^{\prime}\right\}
\end{aligned}
$$

The smoothing $\zeta$ satisfies $\zeta \equiv 1$ at or above the threshold, so that the regularized system reduces to the original system.

Remark 4.2 This is the first analysis of the Bløtekjær system in this complete generality. As noted in the introduction, the use of the fully implicit method of horizontal lines has allowed for the formulation of a precise condition (see Eq. (3.14)) for the local assumption. By using semigroup methods, rather than parabolic methods, we are able to pass to the limit of vanishing heat conduction. The first use of semidiscrete methods in the context of the Kato semigroup framework appears to be in Ref. [17] (see also Ref. [8, Section 7.5]). A weak solution study of the isentropic subsystem in one variable was given in Ref. [20] for the two-carrier model with geometric terms. This alternative approach permits shock formation. Finally, the reader who has followed the arguments of the paper will realize that the case of temperature dependent relaxation terms is elementary, so long as singularity formation is avoided. One can treat a family of such expressions, as long as the functional relationships are smooth. If necessary, one can regularize such expressions as in Eq. (3.3), and proceed much the way we did above. This allows for considerable generality.

\section{References}

[1] Bløtekjær, K. (1970) "Transport equations for electrons in twovalley semiconductors", IEEE Trans. Electron Devices 17, 38-47.

[2] Rudan, M. and Odeh, F. (1986) "Multi-dimensional discretization scheme for the hydrodynamic model of semiconductor devices", COMPEL (Int. J. Comput. Math. Electronic Eng.) 5, 149-183.

[3] Jerome, J.W. (1996) Analysis of Charge Transport: A Mathematical Study of Semiconductor Devices (Springer, Heidelberg).

[4] Baccarani, G. and Wordeman, M.R. (1985) "An investigation of steady-state velocity overshoot effects in Si and GaAs devices", Solid State Electronics 28, 404-416.

[5] Stettler, M.A., Alam, M.A. and Lundstrom, M.S. (1993) "A critical examination of the assumptions underlying macroscopic transport equations for silicon devices", IEEE Trans. Electron Devices 40, $733-740$.

[6] Blatt, F.J. (1968) Physics of Electric Conduction in Solids (McGraw-Hill, New York).

[7] Kato, T. (1975) "Quasi-linear equations of evolution, with applications to partial differential equations", In: Everitt, W., ed, Spectral Theory and Differential Equations Springer Lecture Notes in Mathematics 448, (Springer, Berlin), pp 25-70.

[8] Jerome, J.W. (1983) Approximation of Nonlinear Evolution Systems (Academic Press, New York).

[9] Kato, T. (1984) Perturbation Theory for Linear Operators, 2nd Ed. (Springer, Berlin).

[10] Collatz, L. (1966) Functional Analysis and Numerical Mathematics (Academic Press, New York), (translated by H. Oser).

[11] Lieb, E. and Loss, M. (2001) Analysis, 2nd. Ed. (American Math. Soc., Providence).

[12] Adams, R. (1975) Sobolev Spaces (Academic Press, New York).

[13] Kato, T. (1975) "The Cauchy problem for quasi-linear symmetric hyperbolic systems", Arch. Rat. Mech. Anal. 58, 181-205.

[14] Friedrichs, K. (1944) "The identity of weak and strong extensions of differential operators", Trans. Am. Math. Soc. 55, 132-151.

[15] Friedrichs, K. (1958) "Symmetric positive linear differential equations", Comm. Pure Appl. Math. 11, 333-418.

[16] Jerome, J.W. (2002) "Analytical approaches to charge transport in a moving medium”. Trans. Theory Stat. Phys. 31, 333-366.

[17] Jerome, J.W. (1982) "Quasilinear hyperbolic and parabolic systems: contractive semidiscretizations and convergence of the discrete viscosity method", J. Math. Anal. Appl. 90, 185-206.

[18] Komura, Y. (1967) "Nonlinear semigroups in Hilbert space", J. Math. Soc. Jpn 19, 473-507.

[19] Majda, A. (1984) Compressible Fluid Flow and Systems of Conservation Laws in Several Space Variables Appl. Math. Sc. 53, (Springer, Berlin).

[20] Chen, G.Q., Jerome, J.W., Shu, C.W. and Wang, D. (1998) "Two carrier semiconductor device models with geometric structure and symmetry properties", In: Jerome, J., eds, Modelling and Computation for Applications in Mathematics, Science, and Engineering (Clarendon Press, Oxford), pp 103-140.

Joseph W. Jerome received the Ph.D. degree in Mathematics from Purdue University, in 1966. He was 
visiting Assistant Professor at the Mathematics Research Center, University of Wisconsin, during 1966-1968, and was Assistant Professor at Case Western Reserve University, during 1968-1970. He joined Northwestern University, in 1970, where he has been Professor of Mathematics and Applied Mathematics, since 1976. He has held sabbatical positions at Oxford University, England, 1974-1975, University of Texas, Austin, 1978-1979, and Bell Laboratories, Murray Hill, 19821983. He was visiting scholar at the University of Chicago, in 1985. He received the Distinguished Alumnus Award from Purdue University's School of Science, in 1996. His research interests include applied analysis, numerical analysis, computational electronics, and ion transport in cellular biology. The most recent of his three books, Analysis of Charge Transport: A Mathematical Study of Semiconductor Devices, was published by Springer, in 1996. He edited, for Oxford Press, the volume, Modelling and Computation for Applications in Mathematics, Science, and Engineering, in 1998. 

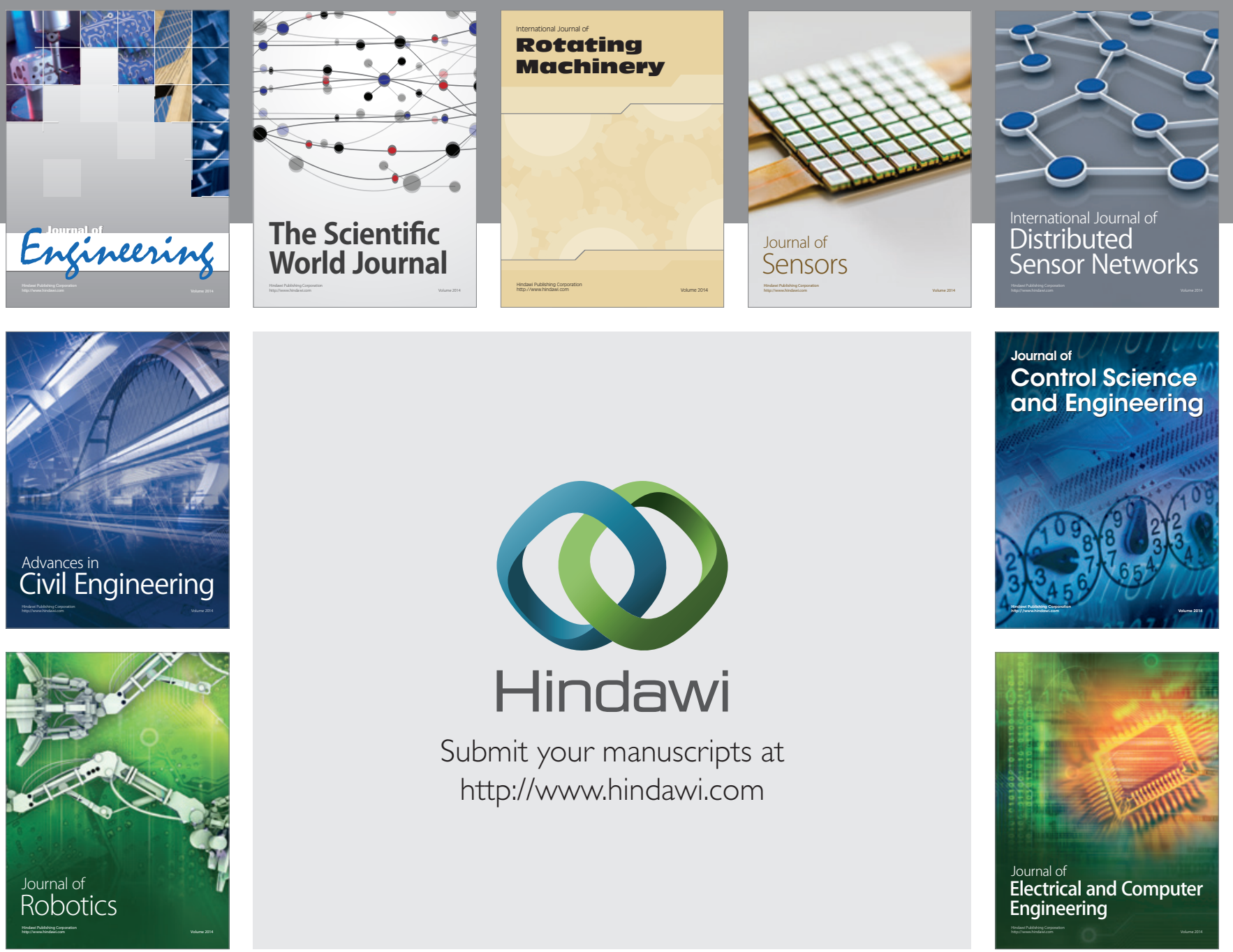

Submit your manuscripts at

http://www.hindawi.com
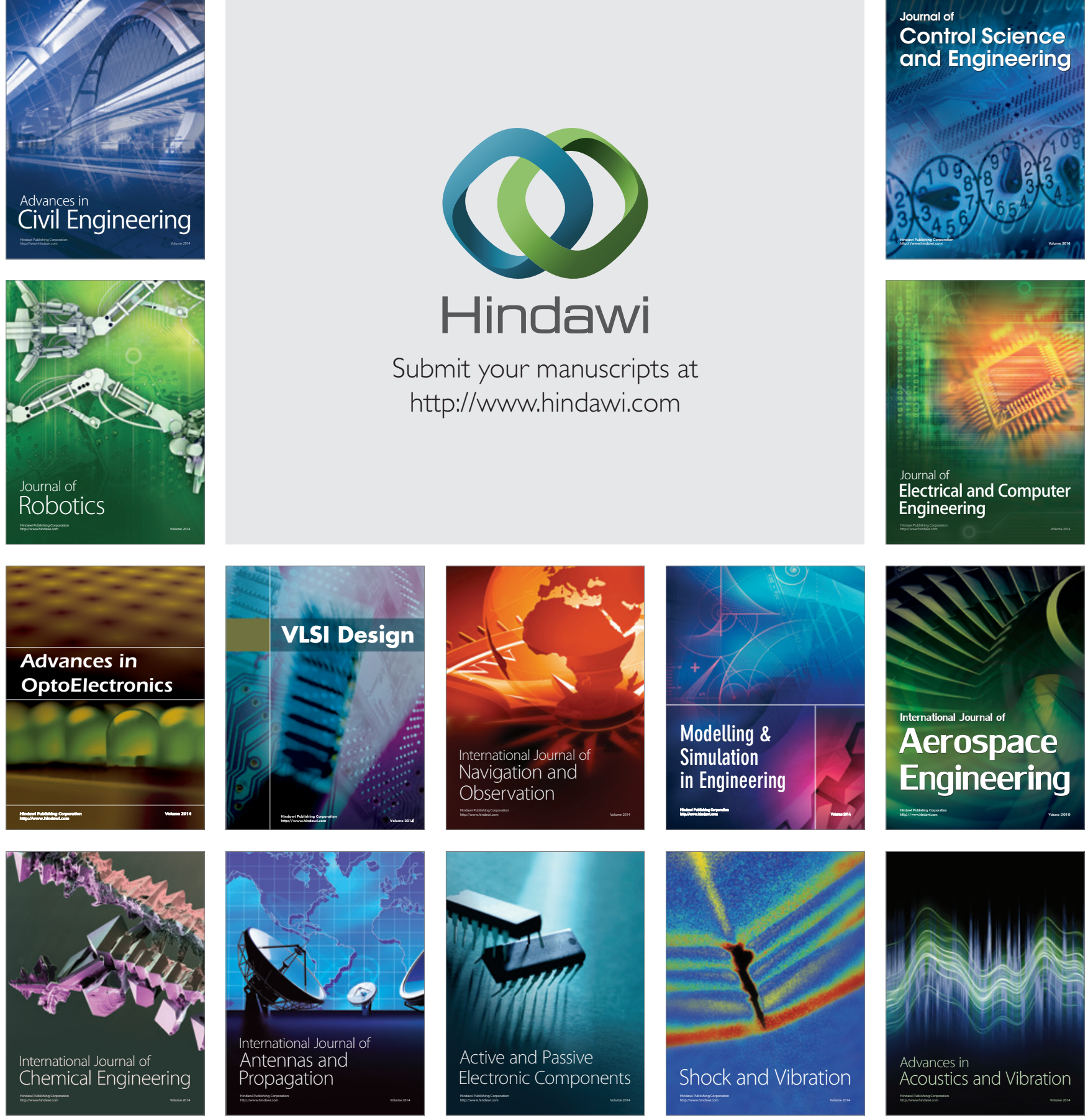